\title{
Hydrogen issue in Core Collapse Supernovae
}

\author{
A. Elmhamdi*, I.J. Danziger ${ }^{\dagger}$, D. Branch ${ }^{* *}$ and B. Leibundgut ${ }^{\ddagger}$ \\ * Osservatorio Astronomico di Trieste, Via G.B.Tiepolo 11 - I-34131 Trieste - Italy \\ $\dagger<$ common address for author 1 and author $2>$ \\ ** Department of Physics and Astronomy, University of Oklahoma, Norman, OK 73019, USA \\ $\$$ European Southern Observatory, Karl-Schwarzschild-Strasse 2. D-85478, Graching - Germany
}

\begin{abstract}
We discuss results of analyzing a time series of selected photospheric-optical spectra of core collapse supernovae (CCSNe). This is accomplished by means of the parameterized supernovae synthetic spectrum (SSp) code "SYNOW". Special attention is addressed to traces of hydrogen at early phases, especially for the stripped-envelope $\mathrm{SNe}$ (i.e. SNe Ib-c). A thin low mass hydrogen layer extending to very high ejection velocities above the helium shell, is found to be the most likely scenario for Type Ib SNe.
\end{abstract}

Keywords: Supernovae:Core Collapse-Spectra-Line identifications-Nucleosynthesis

PACS: 97.60.Bw; 26.30.+k; 95.55.Qf, 95.75.Fg

\section{INTRODUCTION}

Our main goals are to identify traces of hydrogen, helium and oxygen in type Ib-c SNe, and to identify any systematic similarities and differences correlating with other physical properties in the CCSNe family.

We have selected a sample of 45 photospheric spectra of 20 CCSNe objects. In the present paper we show results for a few objects. A detailed analysis for the complete sample has been presented recently in Elmhamdi et al. 2006 [10]. For the purpose of our analysis we make use of the parameterized supernova synthetic spectrum code "SYNOW". The main task is to identify lines and put constraints on the velocities. SYNOW has a number of free input fitting parameters. The most important are: $1 .\left(\tau_{r e f}\right)$, the optical depth of "the reference line". 2. $\left(T_{b b}\right)$ : the underlying blackbody continuum temperature. 3. $\left(v_{p h o t}\right)$ : the velocity at the photosphere, estimated from the match with Fe II lines. The radial dependence of the line optical depths can be chosen to be either exponential with an $e$-folding velocity " $v_{e}$ " as free parameter (i.e. $\tau \propto \exp \left(-v / v_{e}\right)$ ), or a power-law with an index " $n$ " (i.e. $\tau \propto v^{-n}$ ). Detailed description of the code can be found elsewhere $[14,3,10]$

An important aspect to understand within the "SYNOW" line formation logic is the "detachment" concept. When assigning a minimum ion velocity, $v_{\text {min }}$, greater than the photospheric one $v_{\text {phot }}$, the ion is said to be "detached" from the photosphere, and consequently has a "non-zero" optical depth only starting at $v_{\min }$. In "SYNOW" the profile of a detached line has a flat-topped emission, and the absorption minimum is blueshifted by the detachment velocity. An undetached line has a rounded emission peak. A slightly detached line has a flat top but only over a small wavelength interval. These possibilities are illustrated in Fig. 1(left), where the code run adopts $v_{\text {phot }}=10000 \mathrm{~km} \mathrm{~s}^{-1}$ and $\mathrm{T}_{b b}=6000 \mathrm{~K}$, including only hydrogen Balmer lines with $\tau(\mathrm{H} \alpha)=10$ and having 3 

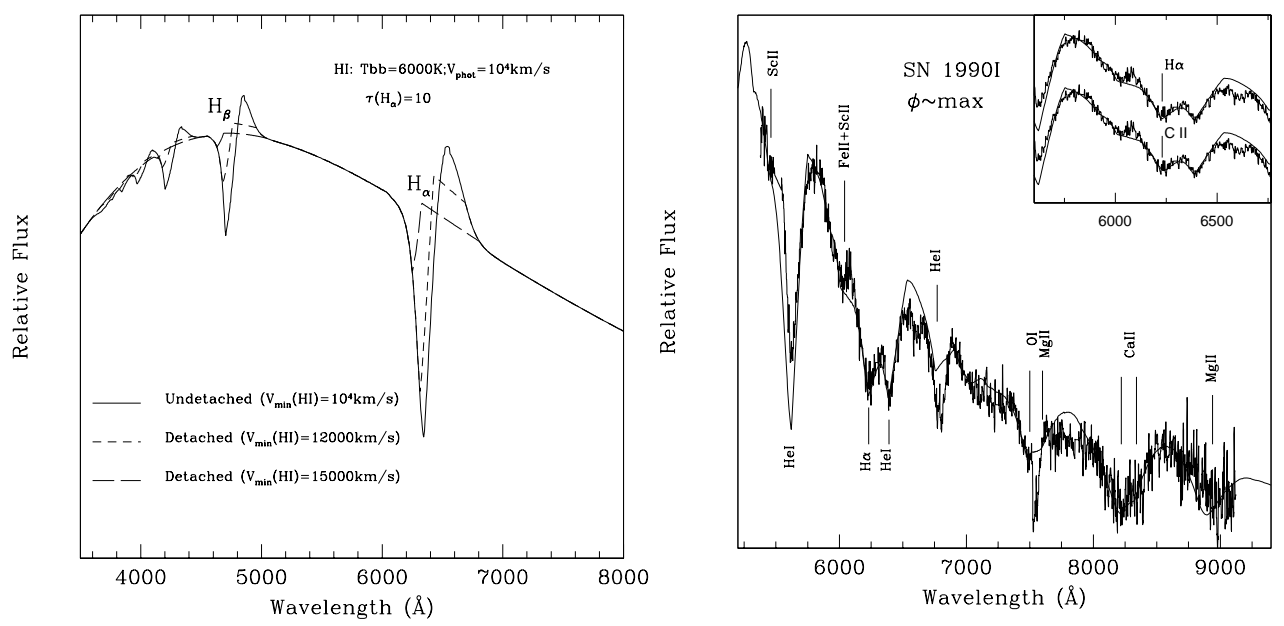

FIGURE 1. Left: the "SYNOW" run for Balmer H I lines, using $v_{\text {phot }}=10000 \mathrm{~km} \mathrm{~s}^{-1}$ and $\mathrm{T}_{b b}=6000 \mathrm{~K}$. The resulted profiles for different detachment velocities are illustrated. Right: The SSp fit, thin line, to the spectrum of SN 1990I near maximum. The region around the $6250 \AA$ trough is zoomed in the window.

different detachment velocities.

We introduce the parameter "contrast velocity", defined as: $v_{\text {cont }}($ line $)=v_{\text {min }}($ line $)-$ $v_{\text {phot }}$. We discuss as well the behaviour of a similar parameter, defined instead as a ratio, i.e. $v_{\text {cont }}^{\text {ratio }}($ line $)=v_{\min }($ line $) / v_{\text {phot }}$.

\section{HYDROGEN ISSUE IN CCSNE}

In the following we present results for some individual objects of the CCSNe sample.

SN 1990I : the event is one of the well sampled and exploited, both photometrically and spectroscopically, objects among type Ib-c SNe[9]. Fig. 1(right) compares the observed spectrum at maximum light to a synthetic spectrum (SSp) that has a velocity at the photosphere $v_{\text {phot }}=12000 \mathrm{~km} \mathrm{~s}^{-1}$ and $\mathrm{T}_{b b}=14000 \mathrm{~K}$. The introduced lines are He I, Fe II, Sc II, Mg II, O I, Ca II and $\mathrm{H} \alpha$. Absorption troughs of P-Cygni He I lines at $5876 \AA, 6678 \AA$ and $7056 \AA$ are evident, $\tau(\mathrm{HeI}) \sim 2.9$ and $v_{\min }(\mathrm{HeI})=14000 \mathrm{~km} \mathrm{~s}^{-1}$, although they are not simultaneously fitted (i.e. their relative strengths). This limitation is faced each time we analyze and fit He I lines using the LTE approach. A more precise analysis requires NLTE treatment as He I lines are probably non-thermally excited by the decay products of ${ }^{56} \mathrm{Ni}$ and ${ }^{56} \mathrm{Co}$ [15]. Apart from $\mathrm{He} \mathrm{I}$ and $\mathrm{H} \alpha$ lines, the remaining lines are undetached. The absorption minimum near $6250 \AA$ is well fitted by $\mathrm{H} \alpha$, with $v_{\min }(\mathrm{H} \alpha)=16000 \mathrm{~km} \mathrm{~s}^{-1}$ and assigned a moderate optical depth of 0.6 . We tested however other plausible alternative identifications to account for a similar feature seen in type Ib SNe, namely Si II 6355 $v_{\text {cont }}(\mathrm{H} \alpha)$ is only $4000 \mathrm{~km} \mathrm{~s}^{-1}$, hence undetached Ne I line is excluded because it is too blue to fit the observed feature. Si II $6355 \AA$ is obviously also too blue. C II $6580 \AA$ remains then a plausible alternative for $\mathrm{H} \alpha$, since its rest wavelength is slightly redder than $\mathrm{H} \alpha$ and therefore has its contrast velocity as a free parameter. In the window of 

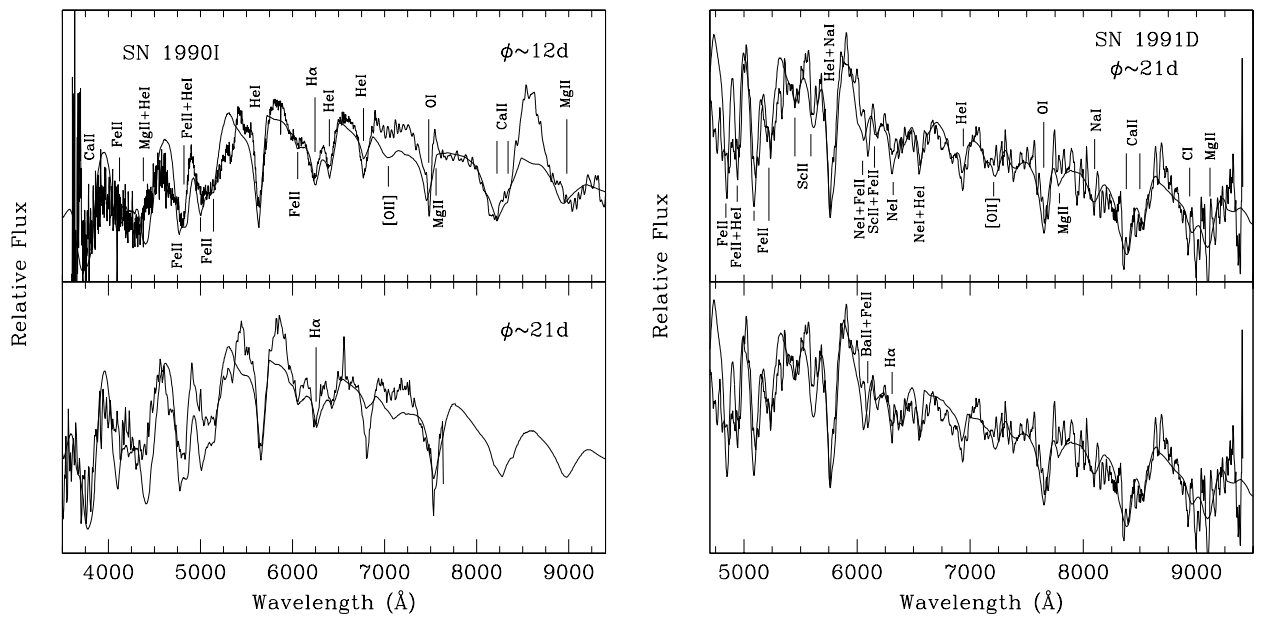

FIGURE 2. Left: SSp fits of SN 1990I spectra at 12d and 21d. Right: SSp fit of SN 1991D around 3 weeks. The top panel illustrates the Ne I possibility, while $\mathrm{H} \alpha$ case is shown in the lower panel.

Fig. 1(right), we show the $\mathrm{H} \alpha$ and C II fit cases. C II, with $v_{\min }(\mathrm{CII})=17000 \mathrm{~km} \mathrm{~s}^{-1}$ and $\tau(\mathrm{CII}) \sim 0.003$, provides a fit as good as H $\alpha$. But an acceptable C II $6580 \AA$ line would require an additional velocity of about $820 \mathrm{~km} \mathrm{~s}^{-1}$ compared to $\mathrm{H} \alpha(\sim 18 \AA$ difference $)$. Furthermore, the He I lines have $v_{\text {cont }}(\mathrm{He} \mathrm{I})=2000 \mathrm{~km} \mathrm{~s}^{-1}$ while $v_{\text {cont }}(\mathrm{CII})=5000 \mathrm{~km}$ $\mathrm{s}^{-1}$. This means that the C II lines would need to be formed above the helium layer, which would be surprising, if not unphysical, in type Ib SNe. SN 1990I presents therefore good evidence for an $\mathrm{H} \alpha$ feature. It is not absolutely clearcut however that $\mathrm{H} \alpha$ is always responsible for the absorption at about $6300 \AA$ seen in type Ib SNe. The presence of the $\mathrm{H} \beta$ Balmer line would be of great support. Unfortunately the optical depth sufficient to fit the $\mathrm{H} \alpha$ trough is so small that the other Balmer features are too weak to be unambiguously detectable ( $\tau(\mathrm{H} \alpha)$ is about 7 times greater than $\tau(\mathrm{H} \beta)$ ). The second factor that makes $\mathrm{H} \beta$ barely discernable is when the contrast velocity of $\mathrm{H} \alpha$ is high as can be clearly seen from Fig. 1(left).

Based on our investigation, the ions that generally might be encountered in shaping the "6000-6500^”" wavelength range are: H $\alpha$, Ne I, C II, Si II, Sc II, Ca I, He I, Fe II, Si I and Ba II. Here, we propose some selection criteria to decide on the presence of hydrogen for the $6300 \AA$ feature:

1 - undetached Ne I $6402 \AA$ line is rejected once its too blue to fit the $6300 \AA$ trough or/and when the other Ne I lines clearly introduce unwanted features. Similar reasoning applies to the Si II $6355 \AA$ line. 2 - with its contrast velocity as a free parameter, C II $6580 \AA$ line could be a candidate for the $6300 \AA$ trough; nevertheless it is ruled out once it exceeds the He I contrast velocity.

The observed spectra at 12days and 21days are compared in Fig. 2(left) to the synthetic ones with $\left(v_{\text {phot }}=10000 \mathrm{~km} \mathrm{~s}^{-1} ; \mathrm{T}_{b b}=5500 \mathrm{~K}\right)$ and $\left(v_{\text {phot }}=9500 \mathrm{~km} \mathrm{~s}^{-1} ; \mathrm{T}_{b b}=5400\right.$ $\mathrm{K})$, respectively. He I is still detached from the photosphere with $v_{\text {cont }}(\mathrm{He} \mathrm{I})=3000 \mathrm{~km}$ $\mathrm{s}^{-1}$ at $12 \mathrm{~d}$ and $v_{\text {cont }}(\mathrm{He} \mathrm{I})=2500 \mathrm{~km} \mathrm{~s}^{-1}$ at 21 days. The He I $7065 \AA$ seems to increase in strength relative to the He I lines at $5876 \AA$ and $6678 \AA$, indicating the non-thermal 

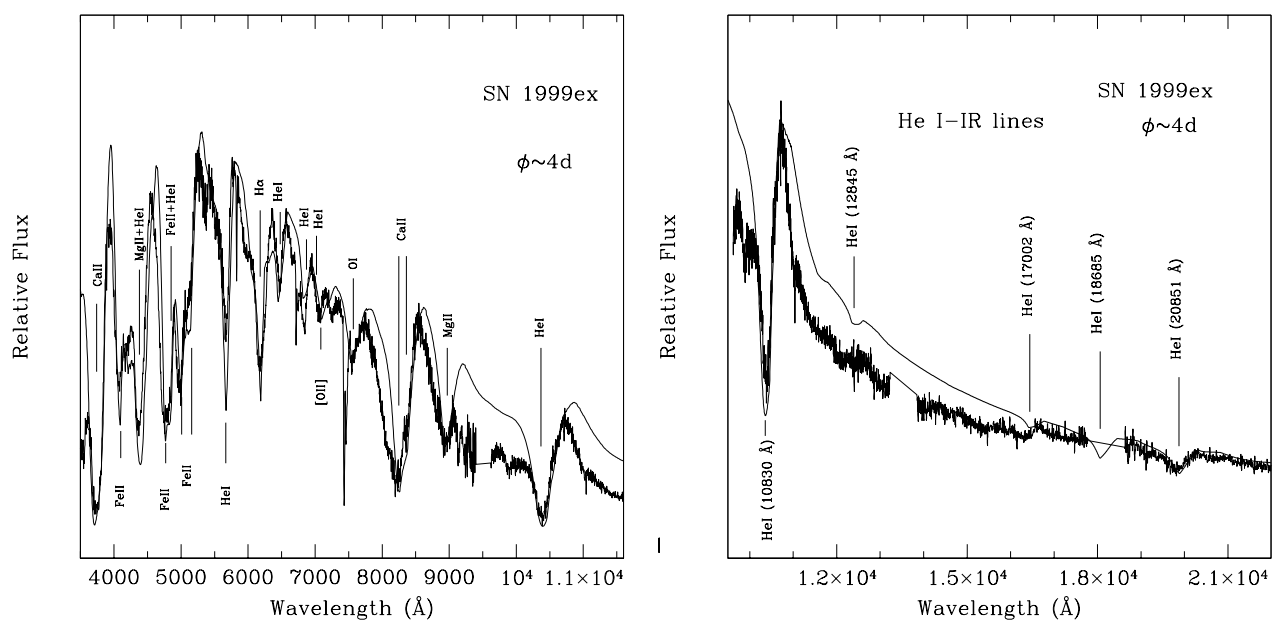

FIGURE 3. Left: SSp fit of SN 1999ex at day 4. Conspicuous line features are indicated. Right: the 4days IR spectrum compared with SSp that contain only lines of He I.

excitation effects are changing but still existent. For the "6000-6500 $"$ wavelength region, we find that $\mathrm{Fe}$ II, $\mathrm{He}$ I and $\mathrm{H} \alpha$ are sufficient to reproduce the overall shape in the SSp at both 12days and 21days. We have, however, tested the Ne I, Si II and C II possibilities as candidates for the $6250 \AA$ trough. They are all ruled out by means of our above criteria. We therefore consider $\mathrm{H} \alpha$ to be the most likely explanation in SN 1990I.

SN $1991 D$ : the event is a peculiar case where its difficult to decide between $\mathrm{H} \alpha$ and $\mathrm{Ne}$ I. The object has narrow features and lower velocities compared to other type Ib events at similar phases [2,3]. Fig. 2(right) illustrates the fit to the 21d spectrum $\left(v_{\text {phot }}=4600 \mathrm{~km} \mathrm{~s}^{-1}\right.$ and $\mathrm{T}_{b b}=7000 \mathrm{~K}=$. The He I lines are evident through our SSp $\left(v_{\text {cont }}(\mathrm{He} \mathrm{I})=1400 \mathrm{~km} \mathrm{~s}^{-1}\right.$ and $\left.\tau(\mathrm{He} \mathrm{I})=1.8\right)$, with the Na I D feature $(\tau(\mathrm{NaI})=4)$ contributing to the He I $5876 \AA$ broad P-Cygni profile. The presence of Na I lines is consistent with the good fit around $8100 \AA$. The undetached Ne I lines possibility is illustrated in the top panel $(\tau(\mathrm{NeI})=2)$, while the $\mathrm{H} \alpha$ case is tested in the bottom panel. Except for adding Ba II lines in order to help the fit at $\sim 6100 \AA$, the other ion parameters are kept unchanged. The fit to the observed absorption near $6300 \AA$ with $\mathrm{H} \alpha, v_{\text {cont }}(\mathrm{H} \alpha)=7400 \mathrm{~km} \mathrm{~s}^{-1}$ and $\tau(\mathrm{H} \alpha)=0.46$, is slightly better compared to the $\mathrm{Ne} \mathrm{I}$ case. However the SSp, in the bottom panel, does not account for the observed features near $6630 \AA$ and $6840 \AA$ as does NeI lines in the SSp of the top panel. Ne I remains hence a strong candidate in this type Ib event.

SNe 1999ex: Because of weak optical He I lines, the object was classified as an intermediate case between $\mathrm{Ib}$ and Ic SNe, while the evident trough at $\sim 6250 \AA$ was attributed to $\mathrm{Si}$ II $6355 \AA$ [12].

In Fig. 3(left) the observed $4 \mathrm{~d}$ spectrum is compared with the best fit that has $v_{\text {phot }}=$ $10000 \mathrm{~km} \mathrm{~s}^{-1}$ and $\mathrm{T}_{b b}=5800 \mathrm{~K}$. To check the consistency of He I identification we combine the IR spectrum with the optical one. In fact assigning the following 

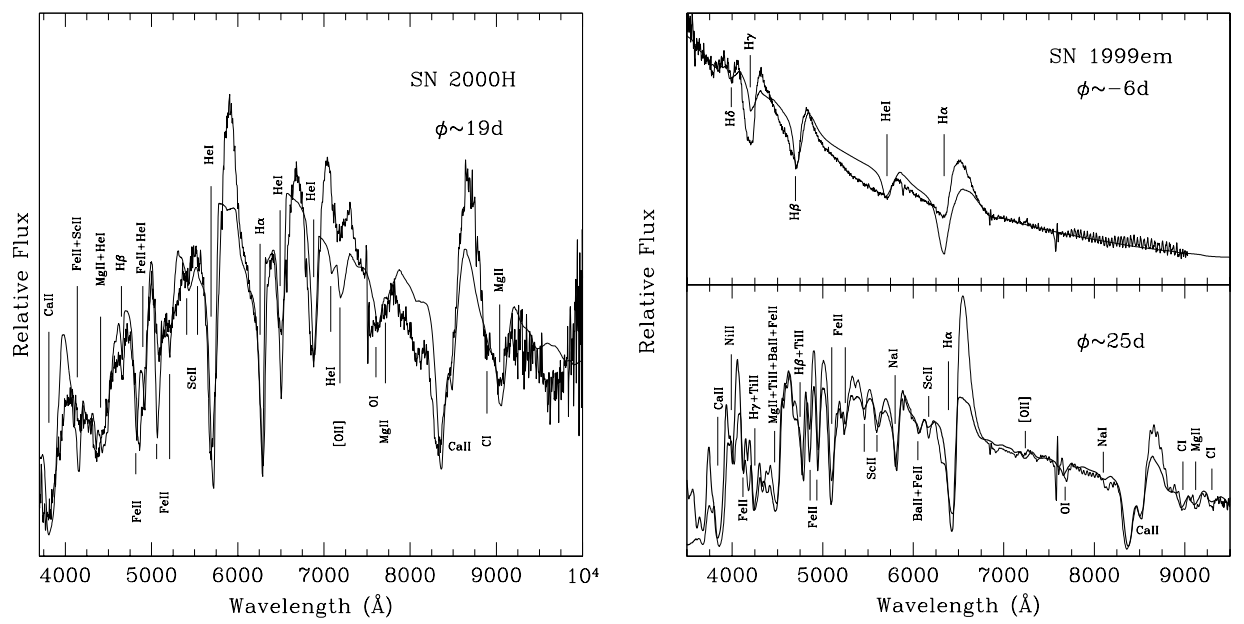

FIGURE 4. Left: SSp fit of SN $2000 \mathrm{H}$ at day 19. Right: SSp fits of SN IIP 1999em observed spectra at day -6 (top) and day 25(bottom). The reported phases are normalized to maximum in type Ib-c SNe.

parameters: $v_{\text {cont }}(\mathrm{He} \mathrm{I})=1000 \mathrm{~km} \mathrm{~s}^{-1}$ and $\tau(\mathrm{He} \mathrm{I})=2.35$, we obtain a good match to the observed He I profiles including the IR He I 10830 ̊. Furthermore, unambiguous evidence for the presence of helium in this event is clear from the extended fit to other He I-IR lines, adopting equal $v_{\min }(\mathrm{He} \mathrm{I})$ and $\tau(\mathrm{He} \mathrm{I})$ as in the optical part of the spectrum (Fig. 3-right). The OI $7773 \AA$ line with $\tau=0.5$, on the other hand, is found to be not so strong and deep as is the case in most type Ic SNe [16]. These two facts point to a Ib nature, more than a Ic class.

As far as the trough around $6250 \AA$ is concerned, we checked the Si II identification attributed by Hamuy et al.(2002)[12]. We test as well the Ne I possibility. The undetached Ne I fit introduces unwanted features in the spectrum. The Si II $6355 \AA$ is rather blue to account for the feature. We note here that if one adopts C II $6580 \AA$, then one needs to assign it a very high velocity, about $8000 \mathrm{~km} \mathrm{~s}^{-1}$ higher than the one of $\mathrm{He}$ I. The most likely identification therefore remains $\mathrm{H} \alpha$. In fact the best fit is achieved using $v_{\text {cont }}(\mathrm{H} \alpha)=8000 \mathrm{~km} \mathrm{~s}^{-1}$ and $\tau(\mathrm{H} \alpha)=1.45$,

SN 2000H : the SN is an example of a type $\mathrm{Ib}$ event with a strong and deep trough near $6300 \AA$ [3]. Fig. 4(left) compares the 19d spectrum with the best fit SSp $\left(v_{\text {phot }}=6000 \mathrm{~km} \mathrm{~s}^{-1}\right.$ and $\left.\mathrm{T}_{b b}=4600 \mathrm{~K}\right)$. Lines of Ca II, [O II], O I, Mg II are seen to develop. The optical He I lines are noticeably deep $\left(v_{\text {cont }}(\mathrm{He} \mathrm{I})=2000 \mathrm{~km} \mathrm{~s}^{-1}\right.$ and $\tau(\mathrm{He} \mathrm{I})=5)$. We note the good match to the $\sim 6250 \AA$ trough with $\mathrm{H} \alpha$, achieved using $v_{\text {cont }}(\mathrm{H} \alpha)=7000 \mathrm{~km} \mathrm{~s}^{-1}$ and $\tau(\mathrm{H} \alpha)=2.5$. No alternative identification to $\mathrm{H} \alpha$, that would be logically acceptable, has been found. In addition, the $\mathrm{H} \alpha$ identification is supported by the presence of the absorption notch near $4660 \AA$, well accounted for by $\mathrm{H} \beta$ in our resulting synthetic spectra.

SN IIP 1999em: Fig. 4(right) displays two spectra during the photospheric phase of the typical type II-Plateau SN 1999em [8]. The phases are normalized to maximum 
light in type Ib-c SNe. The best fit synthetic spectra have $v_{p h o t}=10000 \mathrm{~km} \mathrm{~s}^{-1}$ and $\mathrm{T}_{b b}=10000 \mathrm{~K}\left(-6 \mathrm{~d}\right.$; upper panel) and $v_{p h o t}=4600 \mathrm{~km} \mathrm{~s}^{-1}$ and $\mathrm{T}_{b b}=6400 \mathrm{~K}(25 \mathrm{~d}$; lower panel). At the earliest phase only undetached lines of hydrogen Balmer, He I and a weak contribution from Ca II are sufficient to reproduce the most conspicuous features superimposed on the "hot" continuum. The fit with Na I D, at this phase, is poor compared to He I $5876 \AA$. The He I contribution, in type II SNe, is found to be important shortly after the explosion. In fact in SN 1987A, the He I 5876 feature was clearly present during the first few days, then rapidly faded and disappeared completely around 1 week after the explosion, when Na I D starts to emerge [13]. At -6d, Balmer hydrogen features are evident with $\tau \geq 15$ for $\mathrm{H} \alpha$. The corresponding strong and broad P-Cygni feature cannot be produced completely by the "SYNOW" code. This is because in our SSp treatment we are adopting a resonance scattering source function. At day 25, Fig. 4(right-bottom), $\mathrm{H} \alpha$ is narrower and has $v_{\text {cont }}=1000 \mathrm{~km} \mathrm{~s}^{-1}$ and $\tau=21$. The envelope temperature decreases and many lines emerge at this phase. Apart from the hydrogen lines (slightly detached), all the lines are undetached.

\section{RESULTS AND DISCUSSION}

Based on results presented here and in the larger sample study of Elmhamdi et al. (2006)[10], hydrogen is found to manifest its presence in a different way in the CCSNe sample. SNe of type II and IIb show strong and broad $\mathrm{H} \alpha$ P-Cygni emission component, which is absent in type Ib objects. Indeed, the $\mathrm{H} \alpha \mathrm{P}$-Cygni profile would lose its obvious emission component when it is highly detached. H I Balmer lines have large optical depths, allowing them to be distinctly visible, contrary to normal type $\mathrm{Ib} \mathrm{SNe}$, with the exception of some cases with deep and conspicuous $\mathrm{H} \alpha$ troughs that present the signature of $\mathrm{H} \beta$ as well. Two factors make $\mathrm{H} \beta$ barely discernible in type Ib: the optical depth found to fit $\mathrm{H} \alpha$ is small and the contrast velocity of $\mathrm{H} \alpha$ is high. The opposite is seen in type II and IIb events.

In Fig. 5, upper panel, we report the resulting photospheric velocities from our best fits for the whole CCSNe sample [10]. Data for SN 1987A, corresponding to Fe II $5018 \AA$ absorption, are also shown for comparison (dashed line [17]). The plot indicates the low velocity behaviour of type II SNe, both 1987A and 1999em. SN IIb 1993J follows somewhat similar behaviour as SN Ic 1994I in having higher velocities. Type Ib SNe appear to display a different velocity evolution. The scatter seems to increase at intermediate phases (around 20-30 days). This fact can be simply due to the paucity of available observations outside that range. Around day 20, for example, a scatter as high as $5000 \mathrm{~km} \mathrm{~s}^{-1}$ is measured. SNe 1990I and 1998dt belong to a class with the higher " $v_{\text {phot }}$ ", while objects such as SNe 1991D and 1996aq have the lowest estimated velocities, approaching even type II objects. The remainder of the Ib events follow a similar trend, namely the one described by Branch et al. (2002)[3].

The middle and bottom panels in Fig. 5 display the evolution the of $\mathrm{H} \alpha$ contrast velocities. An increasing trend of " $v_{\text {cont }}(\mathrm{H} \alpha)$ " is found going from type II to IIb to Ib SNe. While in SN IIb 1993J and SN IIP 1999em the line is found to be either undetached or slightly detached, it is highly detached in type Ib events. Moreover, the " $v_{\text {cont }}(\mathrm{H} \alpha)$ " 


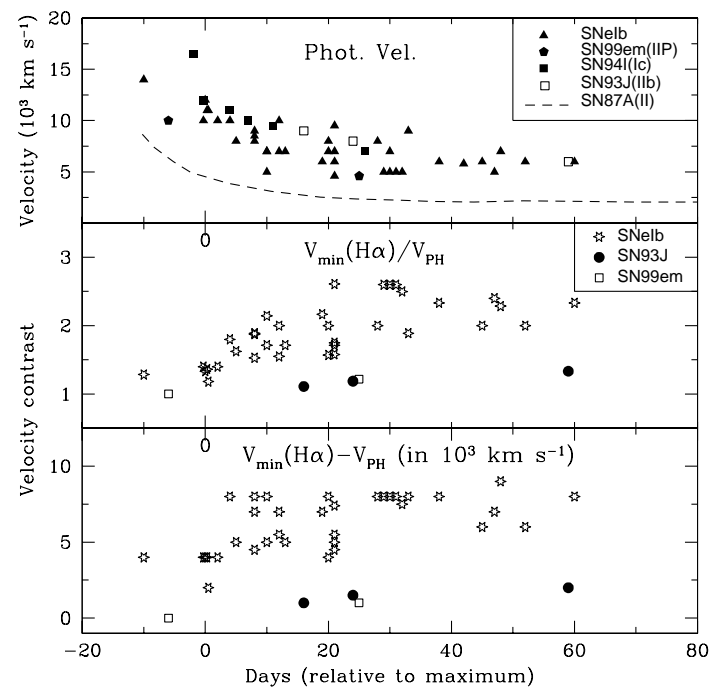

FIGURE 5. The photospheric velocity evolution of the analyzed CCSNe (top). The $\mathrm{H} \alpha$ contrast velocity evolution is also illustrated (middle and lower panels).

is found to increase within the first 15 days, reaching values as high as $8000 \mathrm{~km} \mathrm{~s}^{-1}$, and then follows an almost constant evolution. Up to $\sim 60$ days, type $\mathrm{Ib} \mathrm{SNe}$ are found to have hydrogen down to $11000-12000 \mathrm{~km} \mathrm{~s}^{-1}$. SNe II appear to have hydrogen down to lower velocities ( $\sim 5000 \mathrm{~km} \mathrm{~s}^{-1}$ in SN 1999em). In addition, hydrogen in type $\mathrm{Ib} \mathrm{SNe}$ is found to have very small optical depths independently of the contrast velocity.

An approximate method to estimate the hydrogen mass from the fit results invokes the amount needed to fill a uniform density sphere of radius " $v \times t$ " at an epoch $t_{d}$ since explosion. The required ion mass can be given by ([10]):

$$
M\left(M_{\odot}\right) \simeq\left(2.38 \times 10^{-5}\right) v_{4}^{3} t_{d}^{2} \tau(H \alpha) ;
$$

where $t_{d}$ in days and $v_{4}$ is in $10^{4} \mathrm{~km} \mathrm{~s}^{-1}$. Although non-thermal excitation and "NLTE" effects may be also important for hydrogen, this simple approach seems to give reasonable estimates. For SN Ib 1990 I around maximum light a value of $0.02 M_{\odot}$ is computed. If we adopt a representative $\mathrm{H} \alpha$ optical depth of 0.5 at day 20 with an $\mathrm{H} \alpha$ velocity restriction similar to SN 1990I, the estimated hydrogen mass is of the order 0.015 $M_{\odot}$. Events with higher velocity widths and/or deeper $\mathrm{H} \alpha$ troughs would eject larger amounts. A thin layer of hydrogen, ejected at high velocities down to $11000-12000 \mathrm{~km}$ $\mathrm{s}^{-1}$, appears to be present in almost all the studied type Ib events.

Worth-noticing here is that the presence of $\mathrm{H} \alpha$ in type Ic events was recently readdressed by Branch et al. (2006) [4]. A large type Ic sample study is clearly needed to assess this issue. However, based on these preliminary investigations the progenitor nature of the stripped-envelope SNe should be revised.

An important by-product results of our fit analysis concerns the behaviour of the O I $7773 \AA$ line. At intermediate phases, it seems that type Ib objects tend to have low optical depths in this line, while SNe Ic are found to display the deepest profile. SNe IIb an II, at 
similar phases, are the objects with the lowest O I 7773 $\AA$ optical depth. The stronger and deeper permitted oxygen lines at early phases of SNe Ic spectra might imply that they are less diluted by the presence of a helium envelope. Indeed one might expect oxygen lines to be more prominent for a "naked" $\mathrm{C} / \mathrm{O}$ progenitor core. Despite the paucity of well sampled CCSNe observations, two observational aspects tend to reinforce this belief: First, the forbidden lines, especially [OI]6300, 6364 $\AA$, seem to appear earlier following a $\mathrm{SNe}$ sequence "Ic-Ib-IIb-II". In fact the oxygen line emerges at an age of 1-2 months in Type Ic SN 1987M [11]. SN Ic 1994I displayed evidence for the line at an age of 35-50 days [7]. While in SN Ib 1990I it was hinted at the 70day spectrum [9]. In other Type Ib SNe it appears earlier than in SN 1990I. In SN IIb 1993J, a transition object, the line was visible in the 62day spectrum [1]. In SNe II, however, the line appears later: around day 150 in SN 1987A [5] and after day 138 in SN 1992H [6]. Second, the nebular emission line looks decreasing in breadth following the $\mathrm{SNe}$ sequence above. More investigations concerning the oxygen issue in CCSNe are under progress (Elmhamdi \& Danziger, in preparation).

\section{REFERENCES}

1. Barbon R., Benetti S., Cappellaro E. et al. 1995, A\&AS, 110, 513

2. Benetti S., Branch D., Turatto M. et al. 2002, MNRAS, 336, 91

3. Branch D., Benetti S., Kasen D. et al. 2002, ApJ, 566, 1005

4. Branch D., Jeffery D.J., Young T.R. et al. 2006, "astro-ph/0604047”

5. Catchpole R. M., Whitelock P. A., Feast M. W. et al. 1988, MNRAS, 231, 75

6. Clocchiatti A., Benetti S., Wheeler J. C. al. 1996a, AJ, 111, 1286

7. Clocchiatti A., Wheeler J. C., Brotherton M. S. et al. 1996b, ApJ, 462, 462

8. Elmhamdi A., Danziger I. J., Chugai N. N. et al. 2003, MNRAS, 338, 939

9. Elmhamdi A., Danziger I. J., Cappellaro E. et al. 2004, A\&A, 426, 963

10. Elmhamdi A., Danziger I. J., Branch D. et al. 2006, A\&A,450, 305-330

11. Filippenko A. V. 1997, ARA\&A, 35, 309

12. Hamuy M., Maza J., Pinto P. A. et al. 2002, AJ, 124, 417

13. Hanuschik R. W. \& Dachs J. 1988, A\&A, 205, 135

14. Jeffery D. J. \& Branch D. 1990, in: J.C. Wheeler, T. Piran, \& S. Weinberg (eds.), "Supernovae”, Sixth Jerusalem Winte School forTheoretical Physics (Singapore: World Scientific), 149

15. Lucy L. B. 1991, ApJ, 383, 308

16. Matheson T., Filippenko A. V., Li W., Leonard D. C. \& Shields J. C. 2001, AJ, 121, 1648

17. Phillips M. M., Heathcote S. R., Hamuy M. \& Navarrete M. 1988, AJ, 95, 1087 